doi:10.17659/01.2019.0024

Journal of Case Reports 2019;9(2):95-99

\title{
Encephalopathy due to Phenytoin Toxicity Presenting with a Striking Diurnal Fluctuation in an Elderly Man
}

\author{
Parul Dubey ${ }^{1}$, Carmen Perreira ${ }^{2}$, Varun Pai Kakode ${ }^{3}$, Bhawna Sharma ${ }^{4}$, Raghavendra Sannegowda ${ }^{5}$ \\ ${ }^{1}$ Department of Neurology, Manipal Hospital, Goa; Department of ${ }^{2}$ Medicine and ${ }^{3}$ Neurology, Goa Medical College, Bambolim Goa; \\ ${ }^{4}$ Department of Neurology, SMS Medical College, Jaipur, Rajasthan; ${ }^{5}$ Department of Neurology, Father Muller Medical College and \\ Hospital, Mangalore, Karnataka, India.
}

\section{Corresponding Author:}

Dr. Parul Dubey

Email: drdubeyparul@gmail.com

This is an Open Access article distributed under the terms of the Creative Commons Attribution License (creativecommons.org/ licenses/by/3.0).

Received Accepted

Published

December 18, 2018

May 2, 2019

May 5, 2019

\begin{abstract}
Background: Undiagnosed phenytoin toxicity in elderly may pose dangers of falls and debility which could be otherwise totally averted. Clinicians need to be aware that though easily available, phenytoin is one of the difficult drugs to dose appropriately owing to its saturable metabolism and a narrow therapeutic window. The difficulty is compounded in the elderly, due to compromised metabolism and erratic clearance of the drug. Case Report: We present a case of an elderly man with fluctuating encephalopathy with a striking diurnal variation, ranging from stupor to a conscious and alert state during the course of the day, which turned out to be due to phenytoin toxicity. Conclusion: Phenytoin should be used with caution in the elderly.
\end{abstract}

Keywords: Anticonvulsants, Brain Diseases, Consciousness, Phenytoin, Stupor.

\section{Introduction}

Patients with phenytoin toxicity usually present with complaints of giddiness and imbalance. Occasionally, higher serum concentrations of the drug may cause lethargy, drowsiness or even coma $[1,2]$. However, it is not common to present with stuporous state during the morning and being awake and alert by evening. We describe this case of an elderly man, who was taking phenytoin, without a warranted indication and interestingly presented with a fluctuating sensorium with a remarkable diurnal pattern. This case highlights the significance of keeping in mind the pharmacokinetics and interactions of phenytoin while prescribing the drug, especially so in the elderly, whose metabolism is already physiologically challenged and often receive polytherapy due to associated comorbidities.

\section{Case Report}

An 85 year old hypertensive man presented to the casualty with 15 days history of episodic unconsciousness lasting a few hours daily. The patient's family reported that he was asymptomatic till last 15 days and following his normal routine of getting up at about $6 \mathrm{AM}$ in the morning. He was habitually a light sleeper and would awaken even with any little disturbance or sound. However, since last 15 days he would experience giddiness while going for micturition at 4 AM. These days he was not getting up at his usual time and for the initial five days when the family members would try to wake him up at about 9 AM he would be in deep sleep, not responding despite calling or vigorously shaking; but at about $10 \mathrm{AM}$ he would wake up and would be drowsy for the initial 1-2 hours and thereafter would be conscious and alert throughout the day, carrying out his routine activities. But through the day he did complain of occasional mild giddiness. Over the next one week the family noticed that the duration of his sleep was progressively increasing and he would not respond to repeated calling or shaking during 
his sleep till about the afternoon, but would wake up thereafter and after a few hours of drowsiness would be apparently conscious and alert for the rest of his waking hours. He would eat well and talk normally and carry out his routine daily activities independently. He had not been incontinent in bed during his stupor. However his usual time of going to bed, about $10 \mathrm{PM}$, had remained unchanged.

There was no associated fever, headache, seizures, cough, urinary burning, abdominal pain, loose motions or vomiting. There was no history of any new one sided weakness, facial deviation, slurring of speech, swallowing difficulty, visual disturbances. There was no history of any fall or alcohol intake or intake of any new medications or drugs apart from what he was already taking for the last one year. His past medical history included history of generalized weakness and exertional breathlessness in 2009. On detailed investigations including peripheral smear, iron studies and bone marrow aspiration he was found to have dimorphic anemia secondary to nutritional deficiency which responded well to methylcobalamin and iron supplementation. He also had a history of chronic low backache which was found to be due to compression fracture of D11 vertebra without any myelopathy and was managed conservatively. In 2014 he had a sudden onset right hemiparesis secondary to intra-cerebral hemorrhage [left sided parietal bleed measuring $(2.1 \times 2.8 \times 1.8 \mathrm{~cm})]$. There were no associated seizures. He was managed conservatively at the local primary health centre and was prescribed anti-hypertensive (amlodipine $5 \mathrm{mg}$ twice a day) and prophylactic anticonvulsants (phenytoin $100 \mathrm{mg}$ one tablet in the morning and two tablets at night every day). He had continued with amlodipine and phenytoin for the last one year, including the last fifteen days. During the days of his stupor he would get up late but was given his regular medications by his daughter in law. Due to progressively deepening and prolonging sleeping hours over the last two weeks the patient was admitted initially at a local primary health centre, where the admitting doctor observed the patient and found him to be unresponsive to verbal commands and even deep painful stimuli till about $1 \mathrm{PM}$. After that when he saw the patient in the evening he was found to be conscious and alert. He referred the patient the same evening to our medical college hospital for further evaluation.

On admission at about 8.30 PM in the casualty the patient was conscious and alert and was oriented to time, place and person. His vital parameters were normal. There was a horizontal jerky gaze evoked nystagmus bilaterally, but rest of the cranial nerve examination was normal. Power was normal in all the muscle groups. Deep tendon reflexes were normal. Plantars were flexor bilaterally. Sensory examination was normal. There was no limb ataxia but had mild gait ataxia, he was unable to take a narrow stance or walk tandem. There were no signs of meningeal irritation or asterixis. Respiratory, cardiovascular and per abdominal examination was normal. A CT scan of the head done in the casualty showed a diffuse cortical atrophy with peri-ventricular ischemic changes. No acute bleed or infarct was seen.

After admission the medicine resident doctors observed him and indeed he was unresponsive to verbal stimuli or vigorous shaking, however would moan to sternal rub and supraorbital pressure. During this period his pupils were normal size and reactive to light, he moved all four limbs minimally to deep painful stimuli and plantars were flexor. This continued till about 1 PM the next day. He was found to be drowsy after waking up for about an hour. By afternoon, patient was back to normal sensorium. A clinical diagnosis of metabolic encephalopathy was considered and his work up initiated. On the second day of admission the patient was seen in the evening hours by the neurology resident who found the patient to be conscious but slightly confused. He was oriented to place and person but disoriented to time. He had nystagmus and gait and limb ataxia. 
The next morning the patient was not arousable again.

His routine metabolic profile was hemoglobin: 12.8 gms\%; total blood count 6200/ $\mathrm{mm}^{3}$; differential count: N62\%, L24\%, M10\%, E03\%, B01\%, platelet count: 4.0 lac $/ \mathrm{mm}^{3}$. Renal (blood urea/serum creatinine $30 \mathrm{mg} \% / 0.8 \mathrm{mg} \%$ ) and liver function and serum electrolytes were normal (sodium: $138 \mathrm{meq} / \mathrm{L}$, potassium: $4.3 \mathrm{meq} / \mathrm{L}$, chloride:102 meq/L). Serum calcium was $9.4 \mathrm{mg} /$ $\mathrm{dL}$, serum ammonia was $30 \mu \mathrm{mol} / \mathrm{L}$. Urine routine microscopic examination, chest radiograph and electrocardiogram were normal. CSF examination revealed normal sugar (71 $\mathrm{mg} \%$, corresponding blood sugar $101 \mathrm{mg} \%$ ) and protein $(70 \mathrm{mg} \%)$ and no cells. Electroencephalography revealed diffuse slowing.

Phenytoin toxicity was suspected after reviewing the patient's drugs and presence of nystagmus and gait ataxia which was noted on few occasions. Phenytoin levels came back high 46.48 $\mu \mathrm{g} / \mathrm{mL}$ (normal therapeutic level: 10-20 mcg/ $\mathrm{mL})$. On the third day of admission phenytoin was stopped. No antiepileptics were initiated as there was no history of seizures anytime ever in life and there is no role of prophylactic antiepileptic medications for intra-cerebral hemorrhage. CT scan brain also had not revealed any gliotic area.

After two days of stopping phenytoin a marked improvement was noted. He started getting up earlier in the morning and his ataxia improved. Within five days of stopping phenytoin his sleep pattern had come back to normal and he was no longer confused and ataxic. He was discharged on his routine anti-hypertensive medications.

\section{Discussion}

Phenytoinis an effective and time tested antiepileptic drug which can have a range of toxic effects [1-3]. Our patient also presented with an encephalopathy secondary to phenytoin toxicity fulfilling the WHO UMC causality assessment criteria (certain) [4].
The interesting feature was the striking diurnal fluctuation of his encephalopathic state. He used to be almost stuporous till the afternoon while in the late afternoon and evening hours he used to be conscious and alert. The fluctuation in sensorium was attributable to the dosing schedule and the prolonged half life of phenytoin in this elderly man. The reasons of phenytoin toxicity in our case could be the altered pharmacokinetics and pharmacodynamics of the drug in an elderly patient. Phenytoin is nearly completely absorbed from the gastrointestinal tract but the time to peak levels ranges from 3 to 12 hours depending upon the formulation. It has an oral availability of $>90 \%$ but is slowly absorbed [3]. A very important feature of phenytoin pharmacokinetics of clinical significance is its saturable metabolism at therapeutic doses. So, in the correct sense of the word phenytoin only has an 'apparent half-life' which varies in relation to serum concentration. On an average its half life is 24 hours but can range from 6 hours, at very low concentrations to 60 hours or more at higher drug concentrations [3].

At lower blood levels, metabolism of phenytoin follows first order kinetics (a fixed percentage of the drug is eliminated) but with rising blood levels, even within the therapeutic range, it changes to zero order kinetics (a fixed amount of the drug is eliminated) as the maximum capacity of the liver to metabolize the drug is approached. As a result, the half life of the drug increases significantly and steady states of the drug take much longer to achieve (about 4-6 weeks rather than the usual 1 week) [3,5]. A small increase in dose may result in a disproportionate increase in its plasma concentration and manifest quickly with toxicity. It may be difficult to predict the correct dose for an individual patient without monitoring of serum levels of the drug. This patient was taking his phenytoin doses of $100 \mathrm{mg}$ during the day and 200 $\mathrm{mg}$ before bedtime. The plasma halflife would have increased and the drug concentration would have been the highest during the morning hours when 
he was almost stuporous. As the drug levels would decrease between the doses his consciousness also improved but it kept on progressively worsening with continued drug intake.

In the elderly, phenytoin metabolism may become even more erratic [6,7]. Generally studies show that aging is associated with a progressive decline in phenytoin clearance, presumably as a result of decreased drug metabolizing capacity $[8,9,10]$. Various studies have evaluated the different dosing schedules of phenytoin, once daily versus thrice and twice daily dosing. The half life of phenytoin is compatible with once daily usage as levels are maintained within therapeutic range. The trough concentrations of the drug correspond to the therapeutic effect, while the peak concentrations correspond to the adverse effects. Once daily doses at night with extended release preparations have been found to be associated with stable trough levels but increased peak levels causing increased toxicity [11].

This patient was taking his phenytoin doses of $100 \mathrm{mg}$ during the day and $200 \mathrm{mg}$ before bedtime. We postulate that the plasma half life would have increased and the drug concentration would have been the highest during the morning hours when he was almost stuporous. As the drug levels would decrease between the doses his consciousness also improved but it kept on progressively worsening with continued drug intake. In our case we also want to highlight the fact that the prescription of phenytoin by the general practitioner without a warranted indication (for seizure prophylaxis in intracerebral bleed) in an elderly male was imprudent. Also this man had a history of dimorphic anemia and compression fracture of vertebra which makes phenytoin a wrong choice for an antiepileptic, if at all the drug was needed. In addition, our patient was on amlodipine, which might have contributed to enhance the antiepilepileptic effect of phenytoin $[13,14]$.
It may be inferred that initially smaller phenytoin dosages should be introduced in old patients, and subsequently adjust doses based on clinical response and serum phenytoin levels. Interpretation of the serum drug levels should take into account the possibility of an increase in the unbound fraction (pharmacologically active) of the drug, due to age related decrease in plasma proteins.

\section{Conclusion}

The phenytoin should be prescribed keeping in mind the erratic pharmacokinetics and dynamics of a the drug like phenytoin which is a known enzyme inducer, more so in the elderly with reduced metabolic clearance, in whom it may cause serious side effects which may be hazardous and there remains higher possibility of drug interactions as elderly are bound to have co morbidities and are commonly on polypharmacy.

Contributors: PD: participated in the patients care, and writing of the paper. CP and VPK participated in study conception and writing of the paper. BS and RS participated in revising the manuscript critically. PD will act as guarantor. All authors approved the final version of this manuscript.

Funding: None; Competing interests: None stated.

\section{References}

1. Craig S. Phenytoin poisoning. Neurocrit Care. 2005;3(2):161-170.

2. Bronstein AC, Spyker DA, Cantilena LR Jr, Rumack BH, Dart RC. 2011 annual report of the American Association of Poison Control Centers' National Poison Data System (NPDS): 29th Annual Report. Clin Toxicol (Phila). 2012;50(10):911-1164.

3. Porter RJ, Beldrum BS. Antiseizure drugs. In: Katzung BG, editor. Basic and Clinical Pharmacology. 11 $11^{\text {th }}$ edition. New York (NY): McGraw-Hill; 2009 (Access medicine).

4. The use of the WHO-UMC system for standardised case causality assessment. Accessed from: http://www.WHOUMC.org/graphics/4409.pdf. Accessed on December 18, 2018.

5. Bourgeois BFD. Pharmacokinetics and pharmacodynamics of antiepileptic drugs. In: Wyllie E, editor. The treatment of Epilepsy. $4^{\text {th }}$ ed. Philadelphia: 
Lippincott Williams \& Wilkins; 2006.

6. Pugh MJ, Foreman PJ, Berlowitz DR. Prescribing antiepileptics for the elderly: differences between guideline recommendations and clinical practice. Drugs aging. 2006;23:861-875.

7. Bergey GK. Initial treatment of epilepsy: special issues in treating the elderly. Neurology. 2004;63(Suppl. 4):S40-S48.

8. Gugler R, Manion CV, Azarnoff DL. Phenytoin: pharmacokinetics and bioavailability. Clin Pharmacol Ther. 1976;19(2):135-142.

9. Bachmann KA, Belloto RJ Jr. Differential kinetics of phenytoin in elderly patients. Drugs Aging. 1999;15:235250.

10. Pugh MJ, Foreman PJ, Berlowitz DR. Prescribing antiepileptics for the elderly: differences between guideline recommendations and clinical practice. Drugs Aging. 2006;23:861-875.
11. Bhuvaneshwari S, Chandy S, Kumar S. Prospective, double-blinded, crossover study to determine the equivalence of the serum levels and the peak level toxicity of diphenylhydantoin (EptoinR). Journal of Clinical and Diagnostic Research. 2012;6(5):780-782.

12. Messé SR, Sansing LH, Cucchiara BL, Herman ST, Lyden PD, Kasner SE, CHANT investigators. Prophylactic antiepileptic drug use is associated with poor outcome following ICH. Neurocrit Care. 2009;11:38-44.

13. Kaminski RM, Mazurek M, Turski WA, Kleinrok Z, Czuczwar SJ. Amlodipine enhances the activity of antiepileptic drugs against pentylenetetrazole-induced seizures. Pharmacol Biochem Behav. 2001;68:661-668.

14. Sharma M, Gehlot A, Parashar D. Potentiation of anticonvulsant activity of phenytoin by calcium channel blockers (verapamil and amlodipine) against maximal electroshock seizures in rats. Int J Basic Clin Pharmacol. 2014;3:608-610. 